\title{
Diseño y construcción de un prototipo electromecánico de prótesis transhumeral comandado por movimientos del brazo funcional
}

\author{
Design and construction of an electromecanic prototype of transhumeral \\ prosthesis commanded by movements of functional arm \\ Juan Pablo España Aguilar \\ Facultad de Ingeniería Mecánica, Corporación Universitaria Autónoma de Nariño, Pasto - Colombia. \\ juanpablo1085295@gmail.com
}

\begin{abstract}
Resumen- En el presente artículo se expone el diseño, construcción y experimentación de una alternativa de prótesis electromecánica transhumeral, cuyo objetivo es proveer al paciente de comodidad física y satisfacción psicológica, mediante una nueva alternativa funcional de prótesis. Dentro de las principales funciones que realiza la prótesis, se encuentran la ejecución de actividades de simetría como levantamiento de objetos a dos manos y actividades de apoyo y sujeción, cuyo funcionamiento se basa en la réplica de movimientos de la extremidad superior contralateral por medio de sensores de deflexión y acelerómetros, obteniendo un movimiento en paralelo.
\end{abstract}

Palabras clave - acelerómetro, antropometría, mecanismo de cuatro barras, servomecanismo.

Abstract In this article is exposed the construction, design and testing of an alternative of a transhumeral electromechanical prosthesis, which aims to provide the patient with physical comfort and psychological satisfaction through a new functional prosthetic option. The main functions performed are activities symmetry as lifting objects with both hands and support activities. And whose operation is based on the replica of movement of the other contralateral upper extremity through deflection sensors and accelerometers, obtaining a parallel movement.

Key Word —anthropometry, accelerometer, four-bar mechanism, servomechanism.

\section{INTRODUCCIÓN}

El miembro superior es una de las extremidades más utilizada por las personas, con la cual se desempeña un rol social importante al momento de relacionarse con su entorno; además, es un miembro con el cual se desarrollan actividades necesarias en lo habitual -prensar, agarrar, sostener y trasladar objetos-, razones por las que se constituye en un órgano imprescindible en el cuerpo humano. [1]

La discapacidad por amputación de un miembro superior es una problemática de inclusión social generada por diversas causas, entre las que se encuentran las patológicas, congénitas, conflicto armado, minas antipersona, accidentes de tránsito y laborales [2], las cuales afectan el componente biopsicosocial de las personas que la padecen (baja autoestima, discriminación laboral, incapacidad de realizar tareas básicas).

Una de las alternativas para la rehabilitación de la población con este tipo de discapacidad, consiste en la adquisición de una prótesis; sin embargo, esta opción representa un problema económico a la hora de adquirirla pues las prótesis que circulan en el comercio y que poseen los componentes estético $\mathrm{y}$ funcional necesarios son de alto costo, lo cual constituye una restricción para el paciente de escasos recursos y para las instituciones de salud que las requieren y desean conseguirlas.

Este tipo de prótesis se dividen en activas y pasivas, entre las activas se destacan las mecánicas, mioelectricas e hibridas, el manejo de las primeras se realiza por medio de un cierre voluntario mediante un arnés el cual es sujetado alrededor de los hombros, las miolectricas son prótesis controladas por señales electromiograficas que son creadas por la interacción química en el cuerpo y las hibridas son la combinación de las dos primeras [3]. Estas prótesis al ser funcionales son costosas siendo difíciles de adquirir y, por otra parte, las pasivas son solamente estéticas, por ende, suplen parcialmente el mencionado órgano. 
El interés de este proyecto es innovar, diseñar y construir un prototipo electromecánico de brazo de bajo costo, estético y funcional con el que se pueda realizar actividades básicas comunes con un grado de dificultad menor, compensando la pérdida o limitación de las funciones físicas del miembro perdido y que esté al alcance de la mayoría de los pacientes. Con esta prótesis, las personas atenuaran dicha carencia y obtendrán un beneficio en calidad de vida, inclusión laboral y mejores oportunidades.

\section{CARACTERÍSTICAS DE LA PRÓTESIS}

Esta prótesis es una ayuda técnica para personas carentes del miembro superior, la cual combina la mecánica y electrónica. La parte mecánica está compuesta por: un mecanismo de cuatro barras que se implementa en los dedos con el fin de tener un cierre de la mano que se adapte a la geometría del objeto y pueda realizar distintos tipos de agarre [4](cilíndrico, de pinza lateral, esférico, pinza digital)(Fig.1); los actuadores, son seleccionados de acuerdo a las necesidades del paciente y a la antropometría del miembro superior los cuales le proporcionan la movilidad y un espacio de trabajo más amplio al paciente, la prótesis consta de cinco motores DC (corriente directa) que constituyen el sistema motriz y le proveen 5 grados de libertad como se mira en la figura 2, los cuales le permitirán hacer movimientos de flexión y extensión al brazo, rotación de muñeca, tres movimientos independientes -uno para el dedo pulgar, otro para el índice y medio y, otro para el dedo meñique y anular- y un grado de libertad pasivo adicional en la oposición del dedo pulgar.

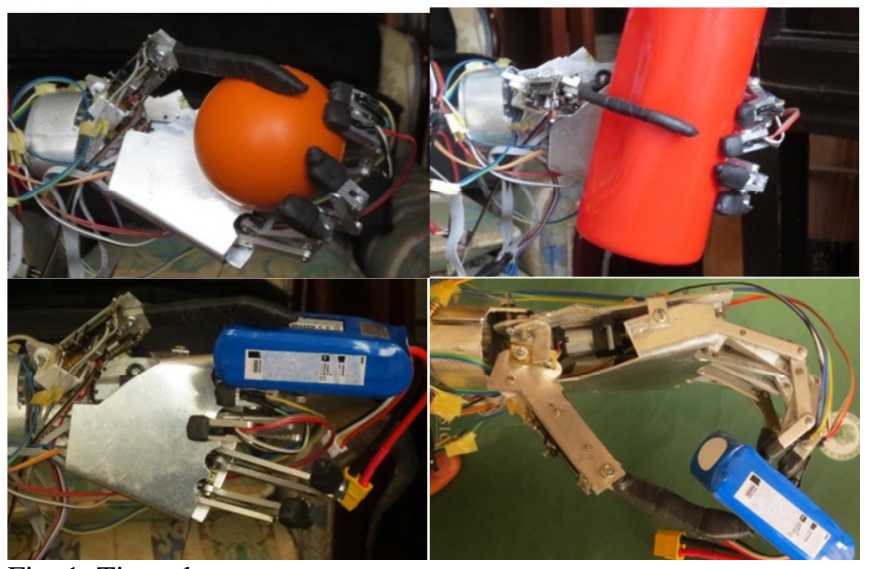

Fig. 1. Tipos de agarre

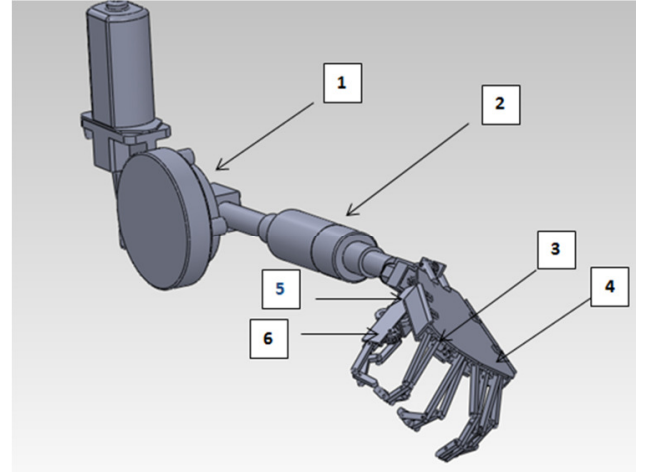

Fig. 2. Diseño de la prótesis en SolidWorks

La parte electrónica se compone de una serie de sensores; un acelerómetro y giróscopo que dará la lectura de inclinación de la articulación del codo y rotación de la muñeca, tres sensores de deflexión ubicados en un guante que medirán el nivel de flexión en las falanges de los dedos y sensores adicionales de proximidad que estarán ubicados en la región anterior del dedo índice y otro en la región posterior del dedo medio de la prótesis, con el fin de detectar un objeto próximo y proceder a cerrar o abrir la mano.

\section{CONTROL DE MOTORES.}

El control de los cinco actuadores lo proporciona un controlador PID (proporcional integral derivativo) que pretende dar una estabilidad calculando la desviación entre un valor medido y un deseado [5], por cada actuador se adaptaron la posición de los motores, obteniendo una buena respuesta de salida.

Los actuadores están controlados y comunicados por medio de una tarjeta de desarrollo arduino mega con el microcontrolador Atmega128 [6]; donde se usan salidas (I/O) por cada motor y se utilizará un puente " $H$ " para manejar la etapa de potencia; de esta forma se suministra la corriente necesaria que requieren los actuadores (Fig. 3).

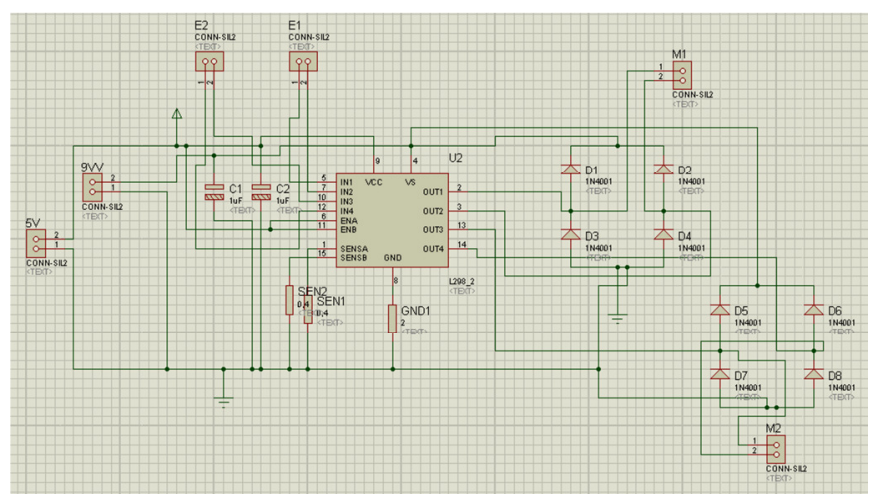

Fig. 3. Esquemático del circuito controlador de motor. 
Para proteger al arduino de posibles ruidos y sobre picos de corriente, se adaptó optoacopladores en medio de la tarjeta arduino y los puentes $\mathrm{H}$ para separar las masas de estos dos circuitos con el fin de evitar daños en la tarjeta [7]. Además se utilizó una batería de litio polímero la cual abastece al circuito de potencia compuesto por los 5 actuadores.

\section{TRANSMISION DE MOVIMIENTO}

El sistema de transmisión del movimiento de los dedos se realiza por medio de pequeños tornillos de potencia, accionado por micromotoreductores. En el actuador principal de la articulación del codo se utilizó un sinfín corona con el propósito de proporcionar torque; así mismo, todos los motores de las articulaciones se escogieron respetando la antropometría del brazo, de tal manera que la prótesis tuviera afinidad con el cuerpo humano.

A. Selección de actuador de articulación del codo.

En la selección del actuador de la articulación del codo se seleccióno un peso de levantamiento máximo de 1,5 kilogramos, este sistema facilmente realiza cualquier actividad que no implique demasiada fuerza, al sostener este peso máximo, los pacientes no sentiran incomodidad en el muñón a diferencia de otras personas con amputación de brazo que expresan molestia y a largo plazo podrían desarrollar afecciones musculoesqueléticas.

Para la selección del motor de la articulacion del codo se tuvo en cuenta la masa y las dimensiones del antebrazo y la masa a levantar, con el fin de obtener el torque requerido por el actuador, a continuacion en la tabla 1 se muestra los datos.

\begin{tabular}{|l|l|}
\hline $\begin{array}{l}\text { Masa de la estructura del } \\
\text { antebrazo y la mano }\end{array}$ & 1,2 kilogramos \\
\hline Masa a levantar & 1,5 kilogramos \\
\hline Masa total & 2,7 kilogramos \\
\hline Factor de seguridad & 1,2 \\
\hline medida antebrazo & $40 \mathrm{~cm}$ \\
\hline
\end{tabular}

Tabla 1. Datos para el calculo de torque.

El torque necesario para levantar un total de 3,24 kilogramos a $40 \mathrm{~cm}$ es:

$$
\begin{gathered}
T=m * d(1) \\
129,6 \mathrm{~kg} . \mathrm{cm}=3,24 * 40
\end{gathered}
$$

Donde $T$ es torque, $m$ masa y, $d$ distancia.

Con el fin de obtener la potencia del actuador se utilizan los siguientes datos.

\begin{tabular}{|l|l|}
\hline Torque requerido & $129 \mathrm{~kg} . \mathrm{cm}=9,33 \mathrm{ft} . \mathrm{lb}$ \\
\hline Velocidad requerida & 20 revoluciones por minuto \\
\hline
\end{tabular}

Tabla 2.Datos para el cálculo de potencia
Con la fórmula de la potencia de motor electrico, se hacen los cálculos y se obtienen los siguientes resultados:

$$
P=\frac{T * n}{c}
$$

Donde " $P$ " es potencia, " $T$ " torque , " $n$ " revoluciones por minuto y " $c$ " factor de conversión igual a 7,04.

$$
\begin{gathered}
P=\frac{9,33 * 20}{7,04} \\
P=26,50 \text { watts }
\end{gathered}
$$

B. Selección de actuador dedos.

La fuerza requerida para una prensión de $12 \mathrm{~N}$, es de 213,26 $\mathrm{N}$ en el punto "O" [8] (Fig. 4) en la cual se la transforma a kilogramos para después obtener un torque en unidades de kilogramo centímetro.

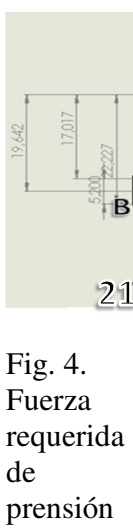

en el mecanismo de cuatro barra

$$
\begin{gathered}
F=m * a(3) \\
m=213,26 / 9,8 \\
m=21,76 \mathrm{~kg}
\end{gathered}
$$

En la Tabla 3 se observan los datos requeridos para el cálculo de fuerza para el tornillo de potencia.

Tabla 3. Datos para el cálculo de tornillo de potencia

$$
T=F * \frac{d m(1+\pi * u * d m)}{2(\pi * d m-u * L)}
$$




$$
T=21,73 * \frac{0,024(1+\pi * 0,05 * 0,024)}{2(\pi * 0,024-0,05 * 0,028)}
$$

$T=3,5 \mathrm{~kg} . \mathrm{cm}$

$\mathrm{T}=3,5 \mathrm{~kg} \mathrm{~cm}=0,25 \mathrm{ft} . \mathrm{lb}$

Velocidad estimada: 150 revoluciones por minuto

$$
\begin{gathered}
P=\frac{T * n}{7,04} \\
P=\frac{0,25 * 150}{7,04}=5,3 \text { watts }
\end{gathered}
$$

\section{SENSORES}

El guante de comando está conformado por un acelerómetro que tiene dos ejes " $\mathrm{x}, \mathrm{y}$ " como se ve en la figura 5 , se utilizó el eje " $\mathrm{x}$ " para la articulación del codo y el eje "y" para la rotación de la muñeca, además de medir la inclinación de estos movimientos, el paciente podrá activar y desactivar la prótesis con un movimiento rotacional rápido de muñeca.

En el guante de comando se colocaron tres sensores de deflexión -uno en el dedo pulgar, otro en el índice y otro en el dedo anular - y, un acelerómetro que mide los movimientos de rotación de la muñeca y extensión y flexión del brazo, como se observa en la (Fig. 5); de otra parte, en los actuadores de la prótesis se situaron potenciómetros que sirven para posicionarlos correctamente y realimentar el proceso. Se utilizó una tarjeta de desarrollo arduino para captar las señales de los sensores y procesarlas.

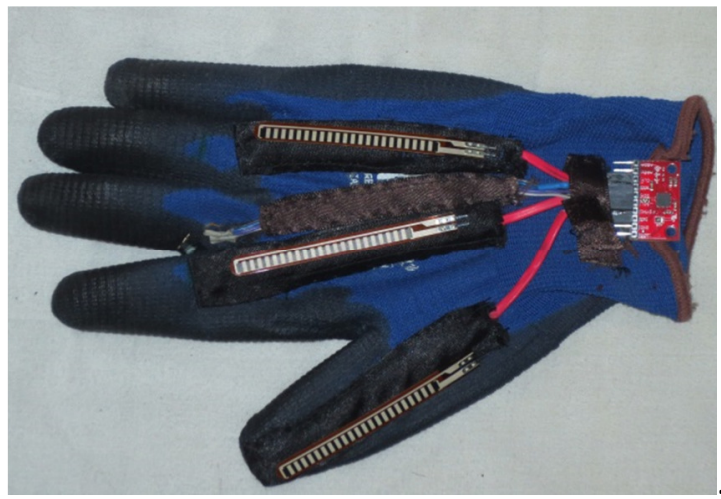

Fig. 5. Guante de control (acelerómetro y sensores deflexion)
Se adaptaron dos tipos sensores adicionales, unos sensores de proximidad ubicados en los dedos de la prótesis (Fig. 6), destinados a hacer un cierre o apertura de mano cuando un objeto este próximo y un guante con tres sensores de deflexión en los dedos para dirigir los movimientos de cierre de la prótesis.

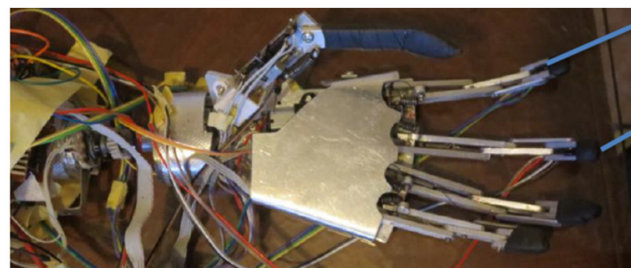

Fig. 6. Sensores de proximidad en el prototipo de prótesis.

\section{ANÁLISIS DE MOVIMIENTO DEL CIERRE DE LA MANO}

Los mecanismos de cuatro barras son generalmente utilizados en sistemas robóticos como también en el diseño de dedos mecánicos los cuales cumple con características

\begin{tabular}{|l|l|l|l|}
\hline Posición & $\begin{array}{l}\text { Angulo } \\
\text { A_B }\end{array}$ & $\begin{array}{l}\text { Angulo } \\
\text { B_C }\end{array}$ & Tiempo \\
\hline 1 & 26 & 360 & 0 \\
\hline 2 & 24,3 & 354,5 & 0,042 \\
\hline 3 & 23,3 & 348 & 0,084 \\
\hline 4 & 19 & 338 & 0,126 \\
\hline 5 & 13,6 & 321,1 & 0,168 \\
\hline 6 & 10,4 & 310,6 & 0,21 \\
\hline 7 & 5 & 297 & 0,252 \\
\hline 8 & 2,7 & 283,6 & 0,294 \\
\hline 9 & $-0,5$ & 276,4 & 0,336 \\
\hline 10 & $-5,5$ & 267,4 & 0,378 \\
\hline 11 & $-10,8$ & 259,1 & 0,42 \\
\hline 12 & -14 & 251,8 & 0,462 \\
\hline 13 & -23 & 239,3 & 0,504 \\
\hline 14 & $-25,6$ & 232 & 0,546 \\
\hline
\end{tabular}

antropométricas y pueden adoptar un movimiento similar al del cierre de la mano.[10]

Con el fin de dimensionar un mecanismo de cuatro barras cruzado que se adapte a la cinemática de un cierre de mano, se captaron fotogramas en un instante determinado (cada 0,042 segundos) (Fig. 7), en los cuales se tomó un registro de ángulos entre las falanges de la mano. (Tabla 4)

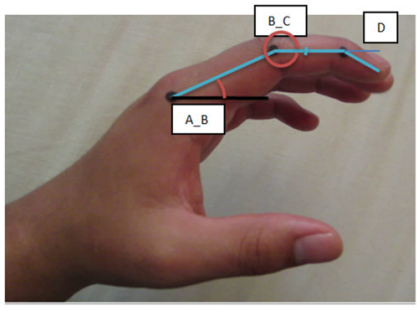

Fig. 7. Fotograma de cierre de mano 


$$
\cos \left(\theta_{1}-\theta_{2}\right)-k_{1} \operatorname{sen} \theta_{1}-K_{2} \operatorname{sen} \theta_{2}-k_{3}=0(5)
$$

Dónde:

$$
\begin{gathered}
k_{1}=\frac{r_{4}}{r_{2}} \\
K_{2}=\frac{r_{4}}{r_{1}} \\
k_{3}=\frac{r_{3}^{2}-r_{1}^{2}-r_{2}^{2}-r_{4}^{2}}{2 r_{1} r_{2}}
\end{gathered}
$$

Se procede a aplicar el método de mínimos cuadrados para obtener un mínimo error.

$$
\boldsymbol{m}=\sum_{i=1}^{n}\left\{\cos \left(\theta_{1 i}-\theta_{2 i}\right)-k_{1} \operatorname{sen} \theta_{1 i}-k_{2} \operatorname{sen} \theta_{2 i}-k_{3}\right\}^{2}
$$

Tabla 4. Relación entre ángulos de las articulaciones de las falanges

En la tabla 4 se relacionan los ángulos B_C ( falange medial) $\frac{\partial M}{\partial k_{1}}=0, \quad \frac{\partial M}{\partial k_{2}}=0, \quad \frac{\partial M}{\partial k_{3}}=0$, y los ángulos A_B (falange proximal) con el propósito de compararla con la resultante del mecanismo de cuatro barras verificando así la fidelidad de movimiento que se puede obtener con éste mecanismo.

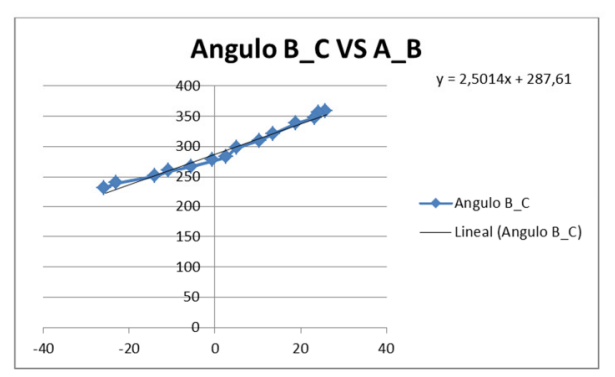

Gráfica 1. Relación entre ángulo de falanges medial y proximal.

\section{a) DIMENSIONAMIENTO DEL MECANISMO DE CUATRO BARRAS CRUZADO}

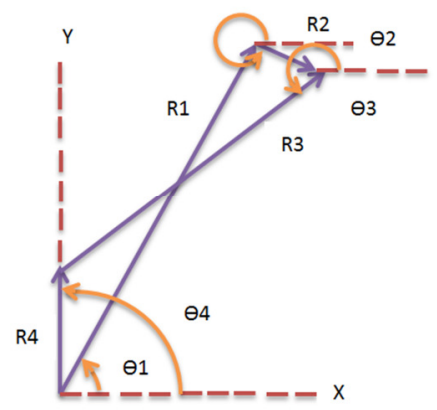

$$
\begin{gathered}
\frac{\partial M}{\partial k_{3}}=2 k_{1} \sum_{i=1}^{n} \operatorname{sen} \theta_{1 i}+2 k_{2} \sum_{i=1}^{n} \operatorname{sen} \theta_{2 i}+2 n k_{3}-2 \sum_{i=1}^{n} \cos \left(\theta_{1 i}\right. \\
\left.-\theta_{2 i}\right)=0
\end{gathered}
$$

Estas tres ecuaciones se las puede plasmar en un conjunto de matrices con el fin de obtener un sistema de $3 \mathrm{x} 3 \mathrm{y}$ proceder a resolverlas por el método Cramer.

$$
\begin{array}{r}
{\left[\begin{array}{ccc}
\operatorname{sen}^{2} \theta_{1 i} & \operatorname{sen} \theta_{1 i} \operatorname{sen} \theta_{2 i} & \operatorname{sen} \theta_{1 i} \\
\operatorname{sen} \theta_{1 i} \cos \theta_{2 i} & \operatorname{sen}^{2} \theta_{2 i} & \operatorname{sen} \theta_{2 i} \\
\operatorname{sen} \theta_{1 i} & \operatorname{sen} \theta_{2 i} & n
\end{array}\right]\left[\begin{array}{l}
k 1 \\
k 2 \\
k 3
\end{array}\right]} \\
=\left[\begin{array}{c}
\operatorname{sen} \theta_{1 i} \cos \left(\theta_{1 i}-\theta_{2 i}\right) \\
\operatorname{sen} \theta_{2 i} \cos \left(\theta_{1 i}-\theta_{2 i}\right) \\
\cos \left(\theta_{1 i}-\theta_{2 i}\right)
\end{array}\right]
\end{array}
$$

Fig 8. Mecanismo de cuatro barras cruzado

Para realizar el dimensionamiento de las 4 barras se utiliza la ecuación de freudenstein[11].

Haciendo las sumatorias respectivas de cada término de la matriz se obtiene: 
196

$\left[\begin{array}{ccc}1,19623494 & 0,511114269 & 0,728335233 \\ 0,511114269 & 7,929240097 & -9,372935121 \\ 0,728335233 & -9,372935121 & 14\end{array}\right]\left[\begin{array}{l}k 1 \\ k 2 \\ k 3\end{array}\right]$
$=\left[\begin{array}{c}1,826020777 \\ -1,564550447 \\ 4,617403864\end{array}\right]$

$$
\begin{aligned}
& k 1=1,195617945 \\
& k 2=0,201123416 \\
& k 3=0,402264994
\end{aligned}
$$

Después de encontrados los valores de las constantes se procede a reemplazarlas en las ecuaciones que relacionan las dimensiones de los eslabones $(\mathrm{r} 1, \mathrm{r} 2, \mathrm{r} 3, \mathrm{r} 4)$ con las constantes (k1, k2, k3).

Dando como parámetro la dimensión del eslabón $r 4=0,92$ se tiene:

$$
\begin{gathered}
r_{2}=\frac{r_{4}}{k_{1}}=\frac{0,92}{1,195617945}=0,769476574 \\
r_{1}=\frac{r_{4}}{k_{2}}=\frac{0,92}{0,201123416}=4,574305764 \\
r_{3}=\sqrt{k_{3} 2 r_{1} r_{2}+r_{1}^{2}+r_{2}^{2}+r_{4}^{2}}=5,019419196 \\
r_{4}=0,92
\end{gathered}
$$

Se escala el mecanismo multiplicando por 0,9 para que éstas medidas sean acordes a las medidas antropométricas de una mano común.

$\mathrm{R} 2=0,69 \mathrm{~cm}$

$\mathrm{R} 1=4,11 \mathrm{~cm}$

$\mathrm{R} 3=4,51 \mathrm{~cm}$

$\mathrm{R} 4=0,82 \mathrm{~cm}$

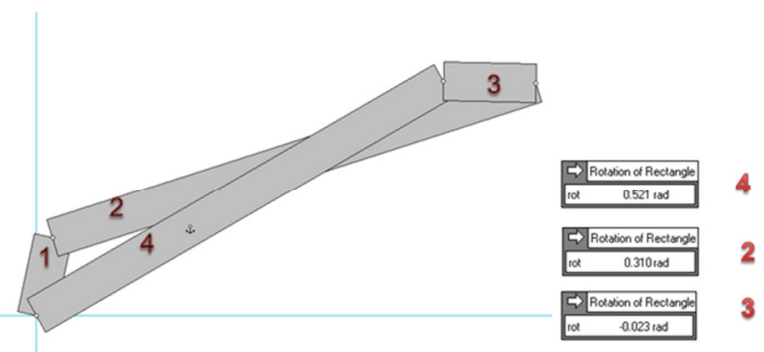

Fig. 9 Mecanismo de cuatro barras simulado en Working Model
Mediante un simulador se procede a verificar los ángulos de catorce posiciones del mecanismo de la barra 4 (A_B) y de la barra 3 (B_C) como se ve en la figura 9.

\begin{tabular}{|c|c|}
\hline $\begin{array}{c}\text { Angulo } \\
\text { A_B_Mecanismo }\end{array}$ & $\begin{array}{c}\text { Angulo_B_C } \\
\text { Mecanismo }\end{array}$ \\
\hline 29,863 & 358,682196 \\
\hline 25,76212 & 346,306297 \\
\hline 21,66124 & 335,477386 \\
\hline 17,56036 & 325,278728 \\
\hline 13,45948 & 315,595733 \\
\hline 9,3586 & 306,485697 \\
\hline 5,25772 & 297,547548 \\
\hline 1,15684 & 289,067765 \\
\hline$-2,94404$ & 280,874462 \\
\hline$-7,04492$ & 272,280087 \\
\hline$-11,1458$ & 264,487855 \\
\hline$-15,24668$ & 256,638326 \\
\hline$-19,34756$ & 249,877419 \\
\hline$-23,44844$ & 242,199778 \\
\hline
\end{tabular}

Tabla 5. Comparación ángulo AB y ángulo BC

Teniendo todos los ángulos A_B y B_C de mecanismo de cuatro barras se procedió a graficarlos para sacar la ecuación de la recta y compararla con la recta de los ángulos A_B y B_C del movimiento de la mano común.

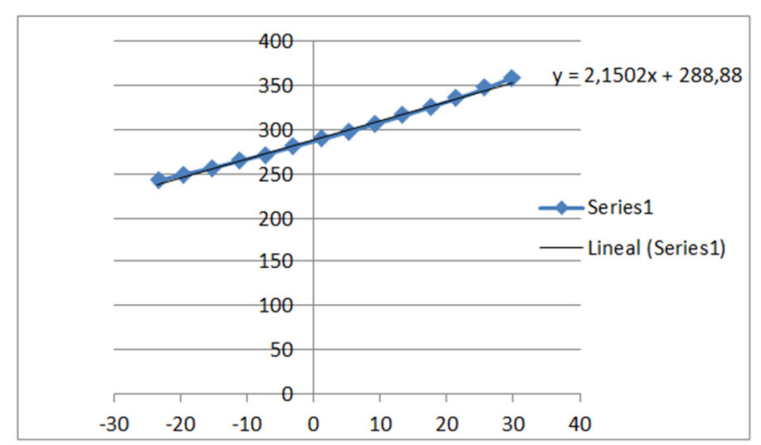

Gráfica 2. Relación entra ángulo de falanges medial y proximal en el mecanismo de cuatro barras.

Se observa que la ecuación de la recta de la gráfica 2 y la gráfica 1 son muy aproximadas, concluyendo que es una buena alternativa.

Con el anterior proceso se dimensionaron los eslabones del primer mecanismo de cuatro barras, lo que representa el 
movimiento de la falange proximal y medial y con un proceso similar se dimensionó el segundo mecanismo de cuatro barras.

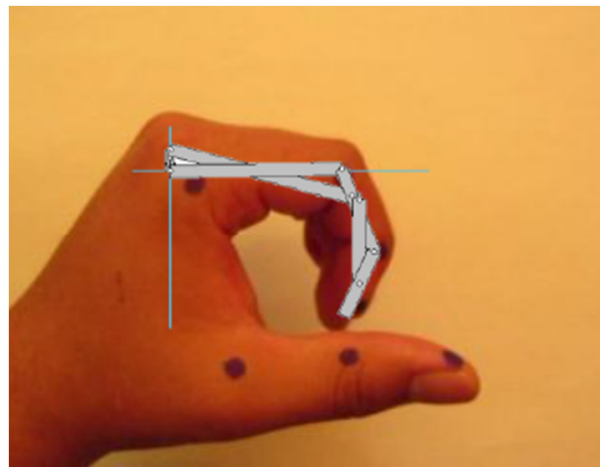

Fig. 10 Superposición del mecanismo de cuatro barras cruzado en la mano.

En la figura 10 se observa la buena respuesta del mecanismo en una posición del cierre de la mano concluyendo una buena alternativa de diseño por medio del mecanismo de 4 barras.

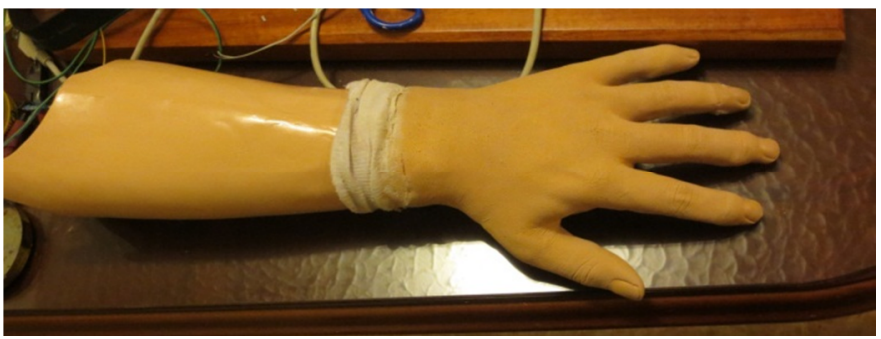

Fig. 11 Prótesis cosmética

Dentro del proceso de investigación se evidenció la necesidad de crear la parte cosmética de la prótesis con materiales como polipropileno para la cubierta del antebrazo y caucho de silicona en la mano (Fig. 11), el proceso de fabricación que se llevó a cabo para darle un aspecto con medidas antropométricas al antebrazo fue el termoformado como se observa en la Figura 12, además se muestra la lámina de polipropileno la cual fue previamente calentada a su temperatura de transición vítrea con el fin de darle forma al antebrazo con ayuda del molde positivo de yeso.

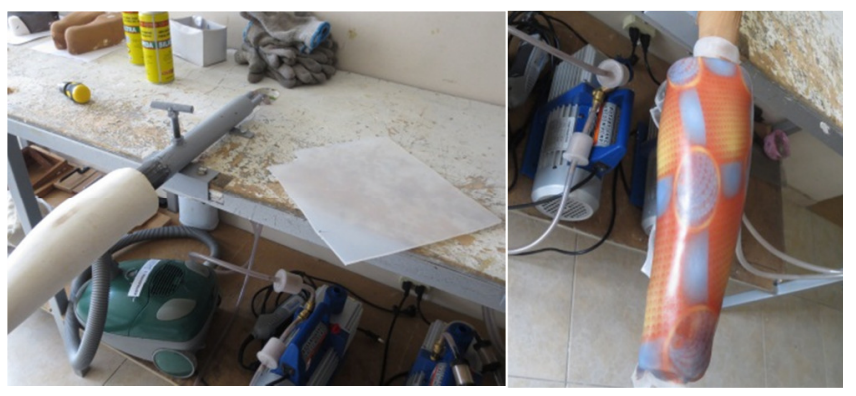

Fig. 12 Proceso de termoformado de la estructura externa del antebrazo

Uno de los elementos principales en la construcción de la prótesis es el encaje (Fig. 13), este permite la fijación del muñón a la prótesis, estos tipos de empalme procuran el contacto entre los estos y están sujetos por medio de correas que le permiten una fijación constante del hombro [12].

\section{PROCESO DE EXPERIMENTACION}

Finalizado el proceso de construcción de la prótesis se la implementó al paciente para que realice la primera prueba, sus reacciones fueron positivas y sin ningún tipo de entrenamiento previo, logró controlar la prótesis de forma sencilla, mostrando satisfacción con la funcionalidad que presentó la misma.

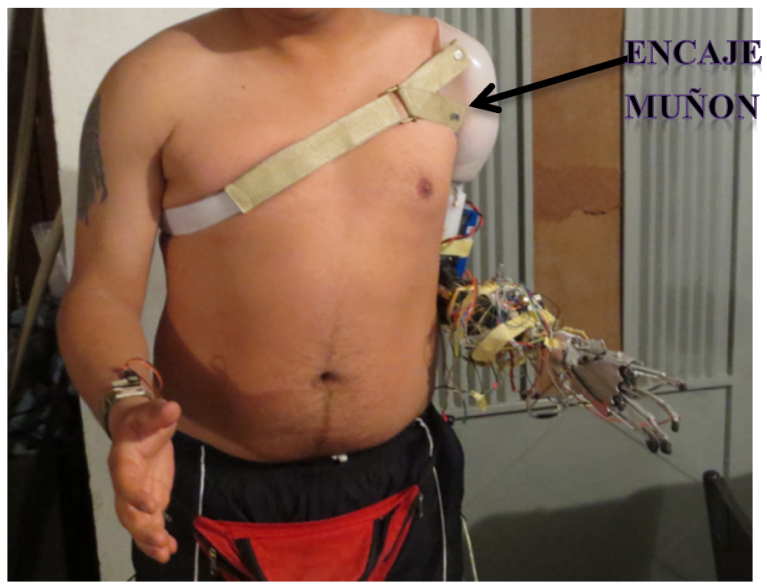

Fig. 13 Paciente con la prótesis transhumeral de brazo

En la figura 14 se muestra el paciente realizando una actividad de prension y se observa que los movimientos de la protesis tienen buena presicion y exactitud, ademas realizò actividades de levantamiento de una bandeja movilizandola de un lado a otro, la apertura de una botella y otras.
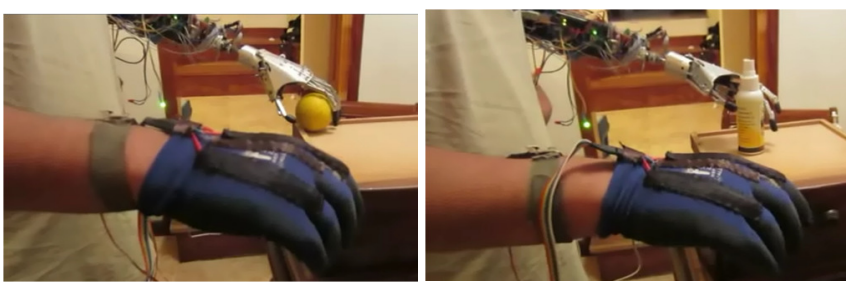

Fig. 14 Prueba de sensores de deflexión con guante de comando

\section{CONCLUSIONES}

La prótesis es un prototipo que cumple las expectativas funcionales, estéticas y económicas del paciente carente del miembro superior; se constituye en una trascendental ayuda para la población en estado de discapacidad pues permite realizar diferentes actividades básicas y relacionarse con su 
entorno laboral, académico, personal y social de forma aceptable, brindándoles bienestar y mejores condiciones de vida tanto físicas como psicológicas.

Se logró la construcción de una alternativa de prótesis con control relativamente sencillo para el paciente, esta consta de un guante que permite manipular la prótesis de forma paralela al comando de la extremidad superior funcional y, además, puede ser desactivada fácilmente. El diseño del mecanismo en los dedos facilita la ejecución de diferentes tipos de agarre y otras actividades, lo cual proporciona mayor comodidad al momento de realizarlas.

Este prototipo de miembro superior, cuenta con la ventaja de hacer uso del mismo sin mayor entrenamiento, donde la característica principal es el movimiento paralelo, dado que el usuario de este prototipo podrá realizar movimientos básicos sin mayor dificultad solo con el uso de un guante en su miembro funcional, estos movimientos son dirigidos por los sensores (acelerómetros) ubicados en la parte posterior del guante y por sensores de deflexión los que conjuntamente determinan el cambio de movimiento.

A partir de los cálculos realizados del mecanismo de 4 barras se concluye que es una alternativa de diseño que cumple con el movimiento de un cierre de una mano común realizando diferentes tipos de agarre y con la fuerza de prensión necesaria.

\section{RECOMENDACIONES}

Dentro de la optimización y mejora del prototipo a futuro se propone una comunicación inalámbrica por módulos bluetooth.

Para obtener un sistema de referencia relativo se puede implementar un segundo acelerómetro en el brazo.

Para mejorar el posicionamiento de la prótesis en la articulación del codo, se recomienda poner el brazo del control paralelo a la fuerza gravitacional.

\section{REFERENCIAS}

[1]. L.Rojas"'Diseño de una prótesis de brazo con seis grados de libertad Bogotá D.C 2009"Trabajo de grado Ingeniero Mecanico.Fundacion Universidad de América.

[2]. L.Aguilar "Optimizacion de la geometría de una prótesis de miembro superior Mexico 2011"Trabajo de grado Ingeniero en control y automatización. Instituto politécnico nacional escuela superior de ingeniería mecánica y eléctrica.
[3]. P.Murillo, I. Luna, A.Mendoza.”Robótica y prótesis inteligentes "revista digital universitaria enero 2004volumen 6 numero 1.

[4]. E. Portilla , R.Piña, O. Avilés ,P.Niño , M. Molina "Diseño del mecanismo actuador de un robot antropométrico" Rev. Fac. Ing. Univ. Antioquia N. ${ }^{\circ}$ 58 pp. 153-162. Marzo, 2011.

[5]. F. Cortes "Arduino Aplicaciones en robótica, mecatronica e ingenierías" editorial Alfaomega 2015.

[6]. F.Cortes "Robótica control de robots manipuladores" editorial Alfaomega,(2011).

[7]. J. Javier , D. García1, F. Melo, J. Chavez "implementación de proceso de pick and place mediante grúa- robot aplicado a celda de manufactura flexible". Revista Scientia et Technica Año XVIII, Vol. 18, No. 4, Diciembre de 2013. Universidad Tecnológica de Pereira

[8]. J. España” Diseño y construcción de un prototipo electromecánico de protesis de brazo izquierdo transhumeral comandado por movimientos del brazo funcional"Pasto 2014. Trabajo de grado Ingeniero mecanico . Corporacion Universitaria Autonoma de Nariño.

[9]. Shigley Diseño en ingeniería mecánica (8. ed). Editorial Mc Graw Hill (2008).

[10]. J. España” Diseño y construcción de un prototipo electromecánico de protesis de brazo izquierdo transhumeral comandado por movimientos del brazo funcional"Pasto 2014. Trabajo de grado Ingeniero mecanico . Corporacion Universitaria Autonoma de Nariño.

[11]. Erdman y Sandor , (1998).Diseño de mecanismos análisis y síntesis ( $3^{\mathrm{a}}$. ed).Editorial Pearson

[12]. H.Barouti, M.Angello, , P.Volckman. Amputaciones de miembro superior, encyclopedia medico quirurgica. Disponible en : http://www.discapacidadonline.com/wpcontent/uploads/2011/05/manual.amputado.miembro. superior.pdf 